\title{
Investigating working practices of dentists on shade taking: Evidence based good practice versus observed practice
}

Shah P 1 , Louca $C^{2}$, Patel R ${ }^{1}$, Fine $\mathrm{P}^{1}$, Blizard R ${ }^{1}$, and Leung $\mathrm{A}^{1}$. 1= UCL Eastman Dental Institute, London, UK

2 = Portsmouth Dental Academy, University of Portsmouth, Portsmouth, UK

\section{$\underline{\text { Abstract }}$}

\section{Introduction:}

The aim of restoring teeth in dentistry is to restore their functionality in the oral cavity and to restore optimal aesthetics. The demand for an optimal aesthetic outcome in dentistry has grown significantly over the past few years. The aim of this study was to investigate UK dentists' working practices on shade taking and to assess whether these clinical practices are in accordance with evidence based guidelines.

\section{Methods:}

A cross-sectional survey was carried out using a questionnaire designed to elicit both quantitative and qualitative data. This included two clinical scenarios (vignettes), which required respondents to describe the steps they would take during shade taking and the prescription they would sent to the dental technician. It was calculated that a minimal sample size of 165 responses was required for this study based on the estimate that $70 \%$ of dentists would comply with good practice guidelines with a 95\% Confidence Interval of $+/-7 \% .200$ questionnaires were distributed to General Dental Practitioners (GDPs), specialists within a university department and postgraduate students studying for a Master's degree in Restorative Dental Practice (RDP) which formed the cohort of respondents.

\section{Results:}

$170(85 \%)$ completed questionnaires were collected and analysed. 167 respondents $(98.9 \%)$ used a manual method for shade taking. 98 (57.6\%; 95\%CI:50\%,65\%) respondents followed the guidelines on the first vignette, 73 (42.9\%; 95\%CI:35\%,51\%) followed the guidelines on 
the second and 41 (26.5\%; 95\%CI:20\%,33\%) followed the guidelines for both. Specialist Professional status $\left(\chi^{2}{ }_{4}=10.76 ; \mathrm{p}=0.03\right)$ and more frequent use of daylight $\left(\chi^{2}\right.$ lin $\left.=6.09 ; \mathrm{p}=0.01\right)$ were both predictive of good practice. However, the number of years qualified of the respondents was not associated with good shade taking practice.

Qualitative data analyses revealed several themes about the quality of clinical shade taking: a) the time shade was taken, b) light source used, c) shades for different aspects of the tooth, d) dental care professional (DCP) opinion, and e) photographic support.

\section{Discussion:}

Within the limitations of this study, it was demonstrated that dentists needed to improve their shade taking techniques in clinical dentistry. The results showed that only $25 \%$ of respondents in this study successfully followed evidence-based good practice guidelines. It also showed that, the many respondents undertook shade taking with a degree of chance and guesswork without consistently following protocols or techniques, which could potentially introduce recording errors and errors in communication with the dental laboratories, resulting in suboptimal clinical outcomes. These findings highlighted the need to incorporate appropriate training on shade taking into the relevant dental curricula. This study also highlighted the importance of compiling accurate and detailed laboratory prescriptions in relation to the shade of the restorations to be fabricated.

\section{Introduction}

The primary aims of restoring teeth are to restore functionality in the oral cavity and to restore aesthetics. The demand for achieving an optimal aesthetic outcome in dentistry has grown significantly over the past few years [1]. The drive towards more aesthetic restorations involves the development of new materials, improvements in the design of tooth preparation, an understanding of colour and improved communication with technicians [2]. Various factors influence the aesthetic outcome of restored teeth such as the shape, contour, texture of the restoration or prosthesis, the colour or shade being pivotal factors [3]. The selection of the correct shade for the restoration is important as it increases the aesthetic appeal of the tooth and enhances the harmonious integration of the natural dentition [4].

Research undertaken between 2006-2016 in respect of shade taking in clinical practice were explored for practical reasons. Two relevant studies were also taken into account, when designing the study, $[5,6]$. 
To understand these issues, it is important to understand colour and its attributes from a dental perspective. Steps used by the clinicians when taking an appropriate shade of a prepared tooth and the adjacent teeth has a significant bearing $[1,4]$ on the final aesthetic outcome of the restoration.

The concept of colour is based on three attributes_[4]:

- Hue: colour tone i.e. red, green, blue and yellow

- Chroma: saturation of purity i.e. strength of the colour

- Value: lightness or darkness of the colour

Furthermore, Translucency, Opalescence and Metamerism [3] are also considered important in this concept.

Fundamentally alterations in the hue, chroma, value of a colour results in the different shades. An understanding of these principles is therefore important for dentists as they encompass the very rudiments of shading taking of teeth [7].

There are three common methods of taking shade of teeth:

- Visual methods, using shade tabs from commercially available shade guides.

- Digital or Instrumental methods, with the help of devices like colorimeter, spectrophotometer or digital cameras, and

- Customised shade tabs, made by laboratories or custom shade matching carried out by the laboratory technician utilising material-specific tabs.

The literature indicated that manual shade taking was the most commonly used method. It also brought into light some recommendations which would enhance the efficacy of manual shade taking $[8,9]$ as follows:

1. Take shade at start of the procedure and after preparation - taking tooth dehydration and eye fatigue into consideration.[10] 
2. Use natural daylight or colour corrected light of 5500 Kelvin (K) in the absence of daylight $[11,12]$

3. Take into account the characterisation of tooth [1]

4. Take into account shade-mapping [1]

These recommendations do not influence other methods of shade taking e.g. technology-based methods. However, technology-based methods have their own limitations in terms of accessibility in mainstream dentistry due to adverse cost implications [15, 16].

However with time and advancement in technology, it could potentially be an inexpensive modern method to enhance colour matching assessment. The introduction of technology into shade taking has brought into question the accuracy of the various techniques now available. Carney \& Johstone (2016) investigated the potential role that Red Green Blue (RGB) data from different shade guides would correlate with their spectroradiometric system and concluded through regression models, that accurate data could be translated from digital images to traditional shade tabs. [17]. The development of technology may also have an impact on inter-observer variability, when taking a shade. Many variables are potential confounding factors, not least of which is the differences in composite materials when using the same target and backing material for the tabs. By developing a 'shade selection programme' 'there could be a reduction in variability in observed selection whilst increasing patient satisfaction' [18].

With the complexities of shade taking there is a genuine need for dentists to be trained in the subtle art of shade taking [6,7]. There is also a need to assess how well information regarding shade of tooth/teeth is conveyed to dental technicians.

Established in 1913 the International Commission on Illumination (Commission Internationale de l'éclairage), was set up to provide information about light, illumination, colour, and colour spaces. This well respected international commission 'is devoted to worldwide cooperation and the exchange of information on all matters relating to the science and art of light and lighting, colour and vision, photobiology and image technology',[19] The international reputation enjoyed by the CIE is pertinent to shade taking in restorative dentistry as much today as it always has been. 
The primary aim of the study was to investigate working practices of general dental practitioners (GDPs) in shade taking. A secondary aim was to assess the effectiveness of relaying shade information from the clinical environment to the dental laboratory.

\section{Methods and materials:}

A questionnaire was employed as the research tool for this study. To help design this questionnaire, a database search using MEDLINE was undertaken. The questionnaire included two evidence based scenarios (vignettes) to test knowledge and compliance. Scoping Sample Size Based on the General Dental Council's register (GDC 2015), there were 41,095 registered dentists in UK. The 'EpiTools Epidemiological calculators' by Ausvet was used for estimation of sample size. Assuming that $70 \%$ of the participants would answer both vignettes correctly, a sample size of 165 was required for this study, estimating that $70 \%$ dentists would comply with good practice guidelines with a 95\% Confidence Interval of $+/-7 \%$. [20].

A pilot study was undertaken with a cohort including GDPs, postgraduate students and teachers, $(n=20)$. Their feedback was used to refine and finalise the questionnaire.

This study was classified as an anonymous service evaluation of an essential practice skill, therefore ethics approval was considered to be unnecessary at the material time. All data were anonymised.

The study was a mixed methods approach, generating both quantitative and qualitative data. The triangulation of data enhanced the validity, reliability and robustness of the findings.

The primary research tool employed for this study was a questionnaire, which contained 17 questions in total including 2 clinical scenarios. The first 15 questions were mainly quantitative in nature. The first few questions gathered relevant demographic information. Subsequent questions adopted a Likert scale type approach. Likert scale is a survey scale that can be used to measure opinions and attitudes of people. Using the 5 point Likert scale in the questionnaire, was aimed to elicit a better understanding of the thought process of the respondents and their understanding of the steps involved in shade taking instead of a binary yes/no answer. The last two questions were clinical vignettes designed to generate qualitative data. The first clinical vignette required the respondents to outline steps they would follow in 
shade taking in clinical practice and the second vignette required them to list the information they would enter on the laboratory prescription regarding the shade of the restoration to be fabricated.

The survey questionnaires were distributed in person to GDPs, newly qualified dentists who were Dental Foundation Trainees (DFTs), Specialist Prosthodontists, dentists in postgraduate education and University Academics involved in the teaching of restorative dentistry. Hard copy questionnaires were distributed by one of the researchers attending places of work of potential respondents. A total of 170 completed questionnaires were returned of the 190 distributed (89.5\%). The answers obtained from the first 15 questions of the questionnaires generated quantitative data that was entered into the SPSS statistical package version 24 [21] for statistical analysis.

The data generated from the clinical vignettes were used for both quantitative and qualitative analysis. A 'good practice' acceptance scale to assess these answers was devised based on opinions from consultants in restorative dentistry and specialist prosthodontists. This scale contained 6 correct answers, with one mark being allocated to each correct answer. The consensus from these specialists formed the basis of the marking criteria. The questionnaire also included enquiries whether dental technician was involved in shade taking. In order to pass the particular clinical vignette, the respondent had to score a minimum of 3 marks (i.e. $50 \%$ ). The respondents needed to individually pass both clinical vignettes to be classified as a pass overall. In order to test the reliability of the scale, 19 questionnaires out of 170 were selected and graded separately using the 'good practice acceptance scale' based on the consensus from the specialists. The scale was found reliable when assessed using Cohen's Kappa scores for inter-observer reliability.

\begin{tabular}{|l|l|l|l|}
\hline Vignettes & \multicolumn{1}{|c|}{ Cohen's Kappa scores } & F value & P value \\
\hline V1 & Cohen's Kappa $=0.69(95 \%$ CI 0.36,0.87) & $\mathrm{F}_{1,18}=5.49$ & $\mathrm{P}=0.000$ \\
\hline V2 & Cohen's Kappa $=0.79(95 \%$ CI 0.55,0.92) & $\mathrm{F}_{1,18}=8.90$ & $\mathrm{P}=0.000$ \\
\hline
\end{tabular}

Table1: Reliability of scoring vignettes by two specialists $(\mathrm{N}=19)$

Kappa values between 0.61-0.80 represent substantial agreement in this context [22]. Table 1 shows that there is substantial inter-observer agreement between the 
specialists. This scale was used to grade all the 170 responses. Statistical Analyses were carried out using SPSS version 24. Descriptive analysis of continuous variables were presented as Mean (SD); Median (IQR). Group comparisons were made by t-test; ANOVA; $\chi^{2}$ as appropriate.

Qualitative data were stored using the 'Framework' spreadsheet method [23], which enabled the data to be analyzed using a thematic approach. Having loaded the qualitative data onto the 'Framework spreadsheet' key themes emerged, which formed the structure of the overall analysis.

\section{Results:}

170 completed questionnaires were collected (85\%). The mean number of years of qualification of the respondents was 13.09yrs. 95 (55.9\%) of the respondents were male; 120 (70.6\%) were UK trained dentists. 167 (98.9\%) indicated they used a manual method for shade taking as opposed to an electronic or a customized approach. $98(57.6 \%)$ of the respondents followed specialists' guidelines on the first vignette, $73(42.9 \%)$ followed the guidelines on the second vignette and $45(26.5 \%)$ followed the guidelines for both vignettes. (Table 2)

\begin{tabular}{|l|c|c|c|}
\hline \multicolumn{4}{|c|}{ Sample size N=170, Frequency (percentage) } \\
\hline Vignettes & V1 (Q16) & V2 (Q17) & V1 and V2 (Q16, Q17) \\
\hline $\begin{array}{l}\text { Pass } \\
\text { Numbers }\end{array}$ & 98 & 73 & 45 \\
\hline $\begin{array}{l}\text { Pass } \\
\text { Percentage }\end{array}$ & $57.6 \%(95 \%$ CI:50\%,65\%) & $42.9 \%(95 \%$ CI:35\%,51\%) & $26.5 \%(95 \%$ CI:20\%,33\%) \\
\hline
\end{tabular}

Table 2: Results of Response to Vignettes V1 and V2

A simple score $(0,1,2)$ was constructed to indicate how many times respondents followed the guidelines. Score 0 meant that the respondents had not passed any of the two vignettes, score 1 meant respondents had passed 1 vignette and score 2 meant that they passed both vignettes. Both Professional status $\left(\mathrm{X}^{2} 4=0.70, \mathrm{P}\right.$ value $\left.=0.03\right)$ and Use of Daylight $\left(X_{\text {Lin }}^{2}=\right.$ 6.090 , $\mathrm{P}$ value $=0.01$ ) showed significant statistical association with faithfully following the guidelines. Number of years of qualification of the practitioner showed no association. 
In relation to the professional status of the respondents, those in possession of postgraduate qualifications achieved a higher pass rate compared to those not in possession of any. Pass rate for specialists was the highest amongst all the categories of respondents. (Figure1).

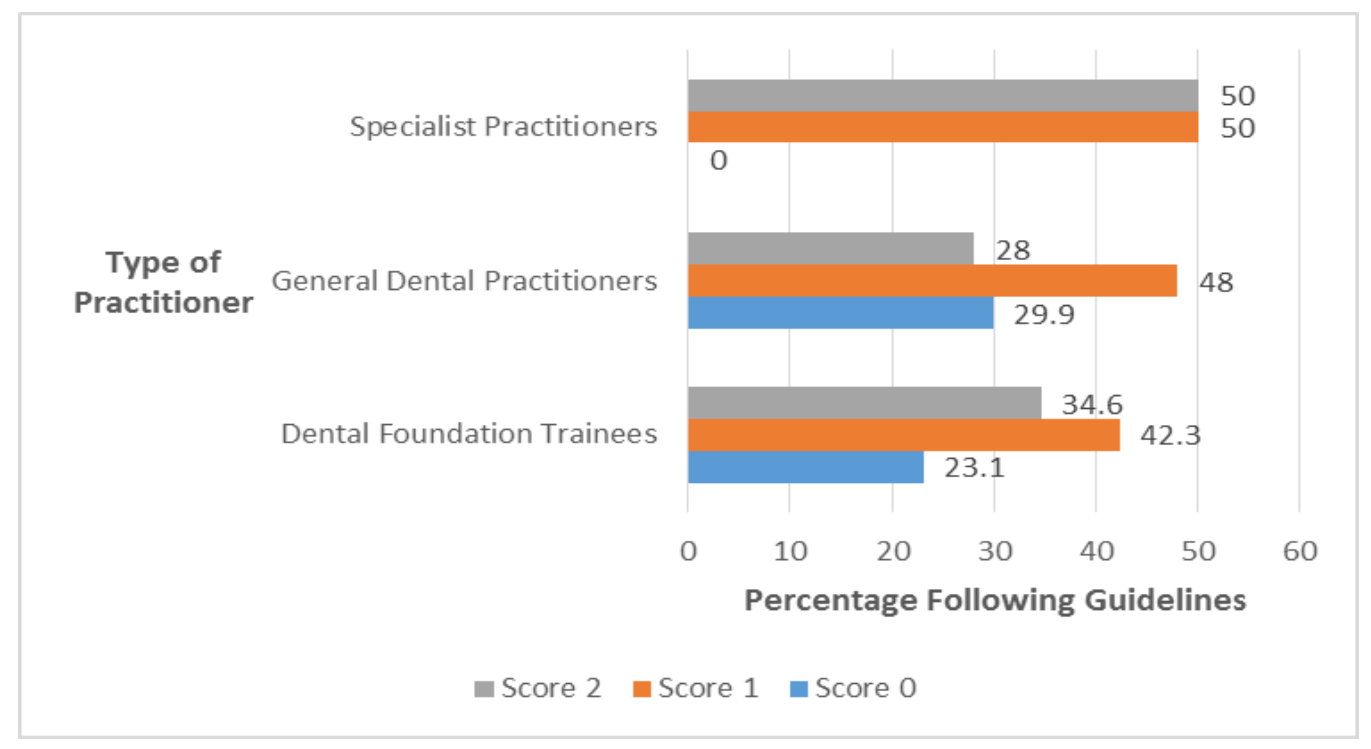

Figure 1: Following the Guidelines: Professional status effect

The results also showed, that respondents who used natural daylight when shade taking had a higher pass rate (Figure2)

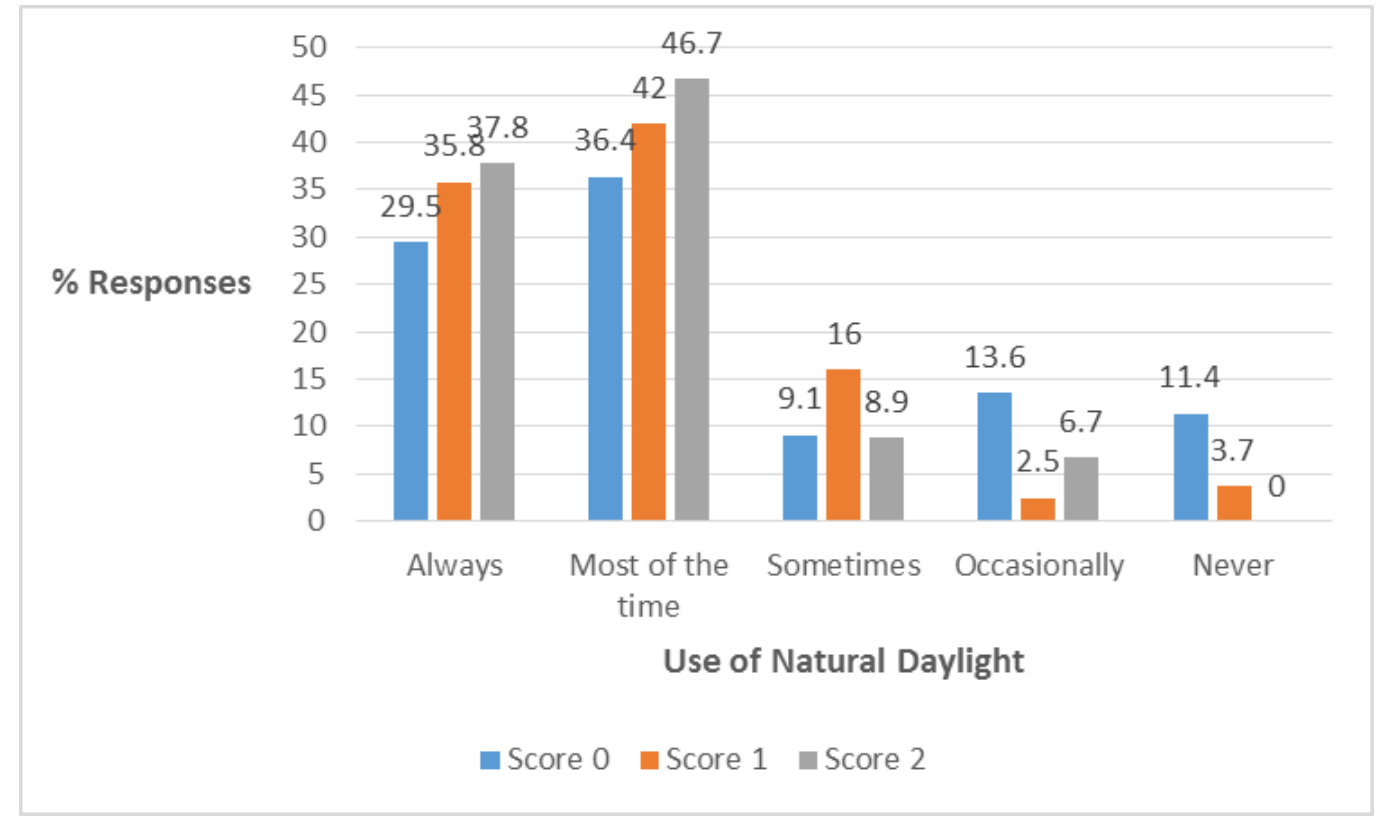

Figure 2: Following the Guidelines: The Use of Natural Daylight 
ANOVA (Analysis of variance) test was undertaken to analyse if the number of years qualified as a dentist affected the pass rate of the respondents.

As illustrated in Table 3, the mean number of years qualified is approximately the same for the respondents who did not pass any, passed 1 or passed both vignettes, therefore the number of years of clinical experience did not affect the pass rate.

\begin{tabular}{|l|l|l|l|l|l|}
\hline $\begin{array}{l}\text { Potential } \\
\text { determinant }\end{array}$ & $\begin{array}{l}\text { Pass =0 } \\
(\mathbf{N = 4 4 )}\end{array}$ & $\begin{array}{l}\text { Pass =1 } \\
\mathbf{( N = 8 1 )}\end{array}$ & $\begin{array}{l}\text { Pass =2 } \\
\mathbf{( N = 4 5 )}\end{array}$ & Test Result & P value \\
\hline $\begin{array}{l}\text { No. of years } \\
\begin{array}{l}\text { Qualified } \\
\text { Mean (SD) }\end{array}\end{array}$ & $\begin{array}{l}13.91 \\
(11.25)\end{array}$ & $13.02(10.3)$ & $\begin{array}{l}13.09 \\
(12.42)\end{array}$ & $\mathrm{F}_{2,167}=0.21$ & $\mathrm{P}=0.81$ \\
\hline
\end{tabular}

Table 3: Number of years qualified in relation to passing the vignettes

The results showed how factors influenced the shade taking procedure in day to day clinical practice. Figure 3, illustrated how the variables considered, influenced shade taking and its impact on the perceptions of the respondents.

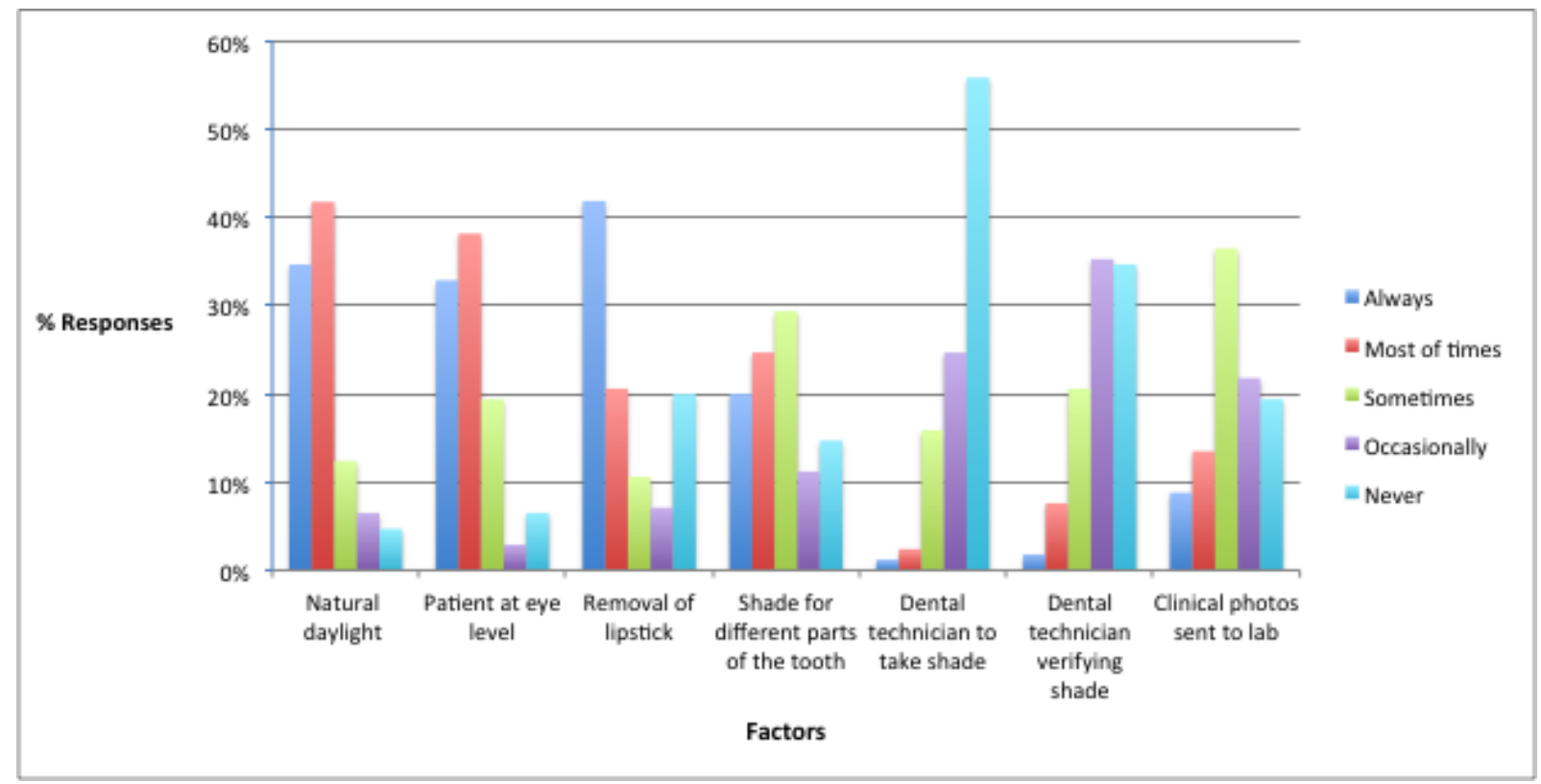

Figure 3: Factors influencing the shade taking: \% Respondents following guidelines 
The main findings were as follows:

- $76.5 \%$ (34.7\% always use, $41.8 \%$ most of the times) of respondents preferred to use natural daylight when taking the shade

- $71.1 \%$ (32.9\% always and 38.2\% most of the times) of respondents preferred to seating patient at eye level when taking the shade

- $62 \%$ of respondents would ask patients to remove their lipsticks when taking the shade

- $25 \%$ of respondents would occasionally leave shade taking to the dental technicians and $56 \%$ of respondents would never leave shade taking to the dental technicians.

Analysis of qualitative data revealed several themes: i) Time the shade was taken, ii) light source, iii) shades for different aspects of the tooth, iv) DCP opinion, v) photographic support and vi) miscellaneous answers. (Table: 3 )

\begin{tabular}{|l|l|l|}
\hline Theme & $\mathbf{N}(\mathbf{\%})$ & Example \\
\hline Time & $77(45)$ & $\begin{array}{l}\text { Assess the shade first thing or } \\
\text { early before dehydration has } \\
\text { occurred }\end{array}$ \\
\hline Suitable Light Source & $75(44)$ & $\begin{array}{l}\text { Patient to stand in natural } \\
\text { daylight by the window }\end{array}$ \\
\hline Shade of Different Parts of & $113(67)$ & $\begin{array}{l}\text { Determine the } \\
\text { lightness/darkness of the } \\
\text { tooth. Then select } \\
\text { hue/chroma using middle 1/3 } \\
\text { of tooth. Then select } \\
\text { enamel/incisal shade }\end{array}$ \\
\hline
\end{tabular}




\begin{tabular}{|l|l|l|}
\hline Another Opinion & $82(48)$ & $\begin{array}{l}\text { Show patient selected shade } \\
\text { with possible options }\end{array}$ \\
\hline Photo Support & $73(43)$ & $\begin{array}{l}\text { Take photos of teeth with } \\
\text { chosen shade tab next to the } \\
\text { tooth }\end{array}$ \\
\hline Miscellaneous & $45(26)$ & $\begin{array}{l}\text { Seat the patient at eye level. } \\
\text { Remove lipstick and use a } \\
\text { neutral colour bib }\end{array}$ \\
\hline
\end{tabular}

Table 3: Qualitative finding of data from the vignettes

It was clear from the above table, that $44 \%$ of the respondents would take the shade at the start of treatment and $45 \%$ of the respondents would do so under natural daylight. $67 \%$ of respondents would take shades of the different parts of the tooth.

\section{Discussions:}

Whilst there are multiple methods of selecting shades, this study indicates that the manual method of shade taking is still by far the most commonly used, with $99 \%$ of the respondents using this method. Efficacy of manual shade taking is highly dependent on the skill and knowledge of the practitioner in the field of shade taking. However it did not appear to be related to clinical experience. It was encouraging to see that more than half the respondents complied with good practice guidelines and passed the first vignette.

However the pass rate dropped for the second vignette, indicating that despite following Specialists compiled evidence-based good practice guidelines, respondents lacked the skills to convey critical information to the laboratory technician, as demonstrated by the low combined pass rate for both vignettes.

The results also indicated that when the bar of comparison was raised to 'good practice acceptance standards', only $25 \%$ of respondents fully complied. A plausible reason for this 
could be, that some respondents might have become complacent and were relying on their years of experience in shade taking rather than being methodical, following all the good practice steps whilst other respondents might need to nurture their confidence $[24,25]$ and competence by enhancing their skills in shade taking.

Several factors have been considered to be important for manual shade taking, and they have influenced the results of this study as follows:

First, at what juncture of a dental appointment is the shade of a tooth/teeth undergoing restorative treatment taken. $90 \%$ of the respondents indicated that they would take the shade before the clinical procedure. This demonstrated that they would take into account the fact that teeth dehydrate during a prolonged period of time with the mouth open. Respondents who mentioned this factor in their answer to the first vignette also scored higher.

\section{Use of Natural daylight}

Second, the use of natural daylight to facilitate shade taking. $75 \%$ of respondents stated that they used natural daylight for shade taking. This demonstrated their awareness that natural daylight $(5500 \mathrm{~K})$ is reported to be the ideal source of light for shade taking [16]. It also demonstrated that 'Metamerism' should be taken into consideration given the fact that there are different sources of light, such as natural light from the window, chair-side light and overhead room light.

Daylight is the ideal light in which to choose a colour. Quality of daylight varies during the day and also throughout the year having a spectrum ranging from $1,000 \mathrm{~K}$ to $20,000 \mathrm{~K}$. An ideal light source should have a full colour spectrum. Other factors that have been investigated that may influence shade matching include the light source, education \& training and gender [16]. This study concluded that natural daylight was the ideal light that can be described as 'North sunlight at noon on a day with very little cloud cover'. Since it is not possible to have ideal natural light, hand held colour corrected light in a spectrum of 5,500 K would be best to do shade selection of teeth.

This study highlighted a strong statistical co-relation between the use of natural daylight in shade taking and passing the vignettes. Qualitative analysis of the data (thematic analysis) also showed that $44 \%$ of respondents referred to the use of natural daylight as the source of light in their answers.

Third, respondents showed an awareness of the importance of taking the respective shades for each part of the tooth, with the understanding that a tooth would not have a single shade 
throughout. Such practice would positively influence the outcome of the shade characteristics of the restoration satisfying both the patient and the dentist.

Fourth, $85 \%$ of the respondents reported carrying out shade taking themselves and did not rely on their technician to do it for them [26]. It was also important that practitioners correctly conveyed all the information to the dental technicians for the fabrication of prosthesis. However, the results of the second vignette, which showed a pass rate of less than $50 \%$ demonstrated a lack, on the part of the respondents, of effectiveness in conveying this information to the technicians. Although practitioners might be carrying out shade taking in line with good practice standards, if they do not communicate fully and effectively with their dental technicians, the aesthetic outcome would be sub-optimal.

Fifth, the use of digital single-lens reflex (DSLRs) cameras in clinical practice has become commonplace [27]. Since the technicians do not usually meet the patient in person, good clinical photographs from DSLRs would help to convey extremely useful information to the technicians.

Only $25 \%$ of the respondents stated that they used clinical photographs to convey information as part of their shade taking protocol. This could be attributed to the fact that the questionnaire did not specify whether the tooth/teeth of concern were in the aesthetic zone. However, if this question in the questionnaire had specified that the tooth/teeth of concern were in the aesthetic zone, more respondents might probably have stated that they would take the help of clinical photos.

Finally, it was interesting to note the wide range of experience of the respondents (1-47 years) [28]. It would have been reasonable to expect that experience in practice might have positively influenced the success rate when scoring the vignettes, however the results indicated no such differences. This may be explained by the fact that recent graduates would probably still follow shade taking methods they were taught at dental schools, whilst more experienced practitioners could possibly be more complacent or have developed their own shade taking routines.

As the vignettes were marked in accordance with a 'good practice acceptance scale' which required all important steps to have been mentioned in the answer to achieve a pass, recent graduates performed well with a $\mathrm{p}$ value of 0.81 , indicating no significance in this finding. With $50 \%$ of specialists having passed both the vignettes as in Figure 1, this study demonstrated that appropriate postgraduate training in restorative dentistry positively influenced the standard of shade taking in clinical dentistry. This may be due to the fact that 
specialists are more knowledgeable in their field of practice, more aware of good practice protocols and are more likely to adhere to them.

It was fascinating to see that pass rate for DFT respondents was $34.4 \%$ compared to $28 \%$ for the GDP respondents in the overall score in the 2 categories. When the bar of comparison was raised, not many GDP respondents could meet the higher standards threshold. It was likely, that as DFT respondents were newly qualified, that they would still follow good shade taking protocols they recently acquired, despite their relative lack of clinical experience .

\section{Conclusion:}

This study demonstrates that the practice of shade taking in restorative dentistry has much room for improvement amongst these respondents. To achieve this, structured training in this interesting and highly relevant area in clinical practice would be highly desirable mandatory in the undergraduate dental curriculum and the relevant postgraduate training programmes. This study highlights the importance of the clinicians writing accurate and detailed laboratory prescriptions in order to convey the necessary information regarding the colour and characteristics of the restorations in full to the technicians. The fact remains, that the practice of shade taking is undertaken by a big majority of respondents in this study, albeit with a degree of guesswork rather than precision and planning for a significant number of them, and therefore much improvement is urgently required, if we are to meet the rightful high expectations of our patients to deliver a high standard of care. Further research in shade taking with view to improving the working practices is warranted. There are also practical and financial implications regarding the procurement of Faculty members at Dental Institutes to help implement effective knowledge transfer [29] in relations to teaching and learning the scope of which is beyond this study. 


\section{Bibliography:}

[1] Magne P, Belser U (2002), 'Bonded Porcelain Restorations in the Anterior Dentition: A Biomimetic Approach', United States: Quintessence Publishing

[2] Aljehani YA, Baskaradoss JK, Geevarghese A, Alshehry MA. 2014. Current Trends in Aesthetic Dentistry. Scientific Research Vol 6:1941-1949

[3] Sikri, V. (2010) 'Color: Implications in dentistry', Journal of Conservative Dentistry, 13(4), p. 249

[4] Chu S.J, Devigus A, Paravina R D, Mieleszko A. J., (2014), Fundamentals of color: Shade matching and communication in esthetic dentistry. United States: Quintessence Publishing (IL)

[5] Glasgow dental School (2012), 'An evaluation into dental shade matching' From:https://dspace.gla.ac.uk/bitstream/1905/494/1/McIlwaine_Simpson_MacPhee_Rolfe. pdf

[6] Habib, S. (2012) 'Awareness of Tooth Shade Selection Principles among dental students, Interns, General Dentist and Specialists'., Pakistan Oral \& Dental Journal Dec Vol. 32 Issue 3, p549-555.

[7] Curd FM, Jasinevicius TR, Graves A, Cox V, Sadan A. (2006), 'Comparison of the shade matching ability of dental students using two light sources', J Prosthet Dent. ;96(6):391-396

[8] (Schropps, 2009; Ragain, 2016) [ Schropps L. 2009. Shade Matching Assisted by Digital Photography and Computer Software, J of Prosthodontics, vol 18(3):235-241

[9] Ragain J. 2016. A Review of Color Science in Dentistry: Shade Matching in the Contemporary Dental Practice. Journal of Dentistry, Oral Disorders \& Therapy, 4(2): 1-5.]

[10] Stevenson, B. (2009), 'Current methods of shade matching in dentistry: A review of supporting literature'., Dental Update; 36: 270-276

[11] Chu, S.J., Trushkowsky, R.D. and Paravina, R.D. (2010) 'Dental color matching instruments and systems. Review of clinical and research aspects', Journal of Dentistry, 38, pp. e2-e16

[12] Burki Z, Watkins S, Wilson R, Fenlon M (2013), 'A randomised controlled trial to investigate the effects of dehydration on tooth colour', Journal of Dentistry 41 pp 250-257 
[13] Bahannan, S.A. (2014) 'Shade matching quality among dental students using visual and instrumental methods', Journal of Dentistry, 42(1), pp. 48-52.

[14] Pottmaier, L.F., Linhares, L.A. and Bernardon, J.K. (2015) 'Comparison between methods visual and instrument for shade matching', Dental Materials, 31, p. e58

[15] Gasparik, C. (2014) 'Influence of Light Source and clinical experience on shade matching.', Clujul Medical, 87(1), pp. 30-33

[16] Clary, J.A., Ontiveros, J.C., Cron, S.G. and Paravina, R.D. (2016) 'Influence of light source, polarization, education, and training on shade matching quality', The Journal of Prosthetic Dentistry.

[17] Carney MN and Johnston WM. A novel regression model from RGB image data to spectroradiometric correlates optimised for tooth colored shades. Journal of Dentistry Vol 51, Aug $2016: 45-48$

[18]. Carney \& Johnston. 2017. The development of a novel shade taking selection program for fixed shade translucent dental materials. Journal of Dentistry Vol 62 July: 81-84

[19]. Davies et al., 1953. Journal of Research of the National Bureau of Standards Vol. 50, No.1, January 1953

[20]. http://epitools.ausvet.com.au/content.php?page=SampleSize

[21] IBM Corp. Released 2016. IBM SPSS Statistics for Windows, Version 24.0. Armonk, NY: IBM Corp. https://wwwo1.ibm.com/support/docview.wss?uid=swg21476197 [22] Landis, J.R. and Koch, G.G. (1977) The Measurement of Observer Agreement for Categorical Data. Biometric, 33, 159-174.

[23]. Ritchie J, and Spencer, L. (1980) Framework, NatCen Learning. https://betterevaluation.org/en/resources/guides/natcen_framework_approach_QDA

[24] Fine, P., Leung, A., Bentall, C., \& Louca, C. (2018). The Impact of Confidence on Clinical Dental Practice. Eur J Dent Educ. doi:10.1111/eje.12415

[25] Fine, P., Louca, C., \& Leung, A. (2017). The Impact of a Postgraduate Learning Experience on the Confidence of General Dental Practitioners. Dentistry Journal, 5 (2), 16. doi:10.3390/dj5020016 
[26] Pravina, R.D. (2011) 'Summary of: Factors influencing the shade matching performance of dentists and dental technicians when using two different shade guides', $\mathrm{Br}$ Dent J, 211(11), pp. 528-529

[27] Ahmad,I. (2009), 'Digital dental photograpt. Part 1: an overview', BDJ 206, April, pp 403-407.

[28] Nakhaei, M., Ghanbarzadeh, J., Amirinejad, S., Alavi, S. and Rajatihaghi, H. (2016) 'The influence of dental shade guides and experience on the accuracy of shade matching', The Journal of Contemporary Dental Practice, 17, pp. 22-26

[29] Davies, B., Leung, A., \& Dunne, S. (2012). So how do you see our teaching? Some observations received from past and present students at the Maurice Wohl Dental Centre. European Journal of Dental Education, 16 (3), 138-143. doi:10.1111/j.16000579.2012.00733.x 